\title{
MASS CONCENTRATION WITH MIXED NORM FOR A NONELLIPTIC SCHRÖDINGER EQUATION
}

\author{
SEHEON HAM
}

(Received 18 May 2011; accepted 16 November 2011; first published online 7 March 2013)

\author{
Communicated by A. M. Hassell
}

\begin{abstract}
This paper is concerned with a mass concentration phenomenon for a two-dimensional nonelliptic Schrödinger equation. It is well known that this phenomenon occurs when the $L^{4}$-norm of the solution blows up in finite time. We extend this result to the case where a mixed norm of the solution blows up in finite time.
\end{abstract}

2010 Mathematics subject classification: primary 42B37; secondary 35B30, 35Q55.

Keywords and phrases: Schrödinger equation, restriction theorem, mass concentration.

\section{Introduction}

We begin with the two-dimensional initial value problem for a nonelliptic nonlinear Schrödinger equation defined by

$$
\left\{\begin{array}{l}
i u_{t}+\square u+\gamma|u|^{2} u=0 \\
u(0, x)=u_{0}(x) \in L^{2}\left(\mathbb{R}^{2}\right)
\end{array}\right.
$$

where $\gamma \in \mathbb{R} \backslash\{0\}$ and $\square=\partial_{x_{1}} \partial_{x_{2}}$. The solution of the linear version of (1.1) (that is, with $\gamma=0$ ) can be written as

$$
e^{i t \square} u_{0}(x)=\int_{\mathbb{R}^{2}} e^{2 \pi i\left(x \cdot \xi-2 \pi t \xi_{1} \xi_{2}\right)} \widehat{u_{0}}(\xi) d \xi .
$$

Note that (1.1) is invariant under the scaling

$$
u\left(t, x_{1}, x_{2}\right) \mapsto(\lambda \mu)^{1 / 2} u\left(\lambda \mu t, \lambda x_{1}, \mu x_{2}\right)
$$

for any $\lambda, \mu>0$. So, we would have to consider rectangles instead of squares when we decompose $\mathbb{R}^{2}$.

It is well known that, in (1.1), there exist maximal existence times $T_{\min }, T_{\max } \in$ $(0, \infty]$ and a unique solution

$$
u \in C\left(\left(-T_{\min }, T_{\max }\right), L^{2}\left(\mathbb{R}^{2}\right)\right) \cap L_{\text {loc }}^{q}\left(\left(-T_{\min }, T_{\max }\right), L^{r}\left(\mathbb{R}^{2}\right)\right)
$$

(C) 2013 Australian Mathematical Publishing Association Inc. 1446-7887/2013 \$16.00 
for any admissible pair $(q, r)$. Recall that $(q, r)$ is called an admissible pair for (1.1) if $q, r \geq 2,1 / q=1 / 2-1 / r$ and $(q, r) \neq(2, \infty)$. Also $\|u(t)\|_{L^{2}\left(\mathbb{R}^{2}\right)}=\left\|u_{0}\right\|_{L^{2}\left(\mathbb{R}^{2}\right)}$ for all $t \in\left(-T_{\min }, T_{\max }\right)$, regardless of $\gamma$. However, unlike the case of the Schrödinger equation, we do not know whether this nonelliptic equation has a blow-up solution related to a given initial datum.

In [12], Rogers and Vargas proved that if $\|u\|_{L_{t, x}^{4}\left(\left[0, T_{\max }\right) \times \mathbb{R}^{2}\right)}=\infty$ for some $T_{\max }<\infty$, then

$$
\limsup _{t \nearrow T_{\max }} \sup _{\substack{\text { a rectangle } R \\|R| \leq T_{\max }-t}} \int_{R}|u(t, x)|^{2} d x>\varepsilon
$$

where $\varepsilon$ is a positive constant depending only on $\gamma$ and $\left\|u_{0}\right\|_{L^{2}\left(\mathbb{R}^{2}\right)}$. When $\|u\|_{L_{t, x}^{4}\left(\left(-T_{\min }, 0\right] \times \mathbb{R}^{2}\right)}$ blows up, there is also a result similar to (1.2). In this note, we shall show that there is also a mass concentration phenomenon for (1.1) when the mixed norm $\|u\|_{L_{t}^{q} L_{x}^{r}}$ blows up in finite time.

In the elliptic case, Bourgain [2] proved the mass concentration phenomenon for an $L^{2}$-critical nonlinear Schrödinger equation with spatial dimension two. This result was extended to higher-dimensional cases by Bégout and Vargas [1]. They made use of bilinear extension (adjoint restriction) estimates for the paraboloid due to Tao [14] in order to get a refinement of the Strichartz estimate which is an essential ingredient in their argument. Moreover, the case where a mixed norm $L_{t}^{q} L_{x}^{r}$ of the solution blows up is considered in [4]. In this case, they utilize a mixed-norm generalization of the bilinear extension estimates for the paraboloid due to Lee and Vargas [10]. A similar result for the higher-order Schrödinger equation, $i u_{t}+(-\Delta)^{\alpha / 2} u= \pm|u|^{2 \alpha / d} u$, can be found in [5].

Our result may be stated as follows.

THEOREM 1.1. Let $(q, r)$ be an admissible pair with $q \leq r \leq 6$. Also let $u$ be the solution to (1.1). If $\|u\|_{L_{t}^{q} L_{x}^{r}\left(\left[0, T_{\max }\right) \times \mathbb{R}^{2}\right)}=\infty$ for some $0<T_{\max }<\infty$ and $\|u\|_{L_{t}^{q} L_{x}^{r}\left([0, t] \times \mathbb{R}^{2}\right)}<\infty$ for all $t \in\left(0, T_{\max }\right)$, then

$$
\limsup _{t \nearrow T_{\max }} \sup _{\substack{\text { a rectangle } \\|R| \leq T_{\max }-t}} \int_{R}|u(t, x)|^{2} d x>\varepsilon
$$

where $\varepsilon$ is a constant depending only on $\gamma$ and $\left\|u_{0}\right\|_{L^{2}}$.

The proof of Theorem 1.1 basically follows the argument of Rogers and Vargas [12] which was partially based on a modification of the method of Bougain [2] and some new ideas essential for handling the hyperbolical situation. In the same manner, decomposing $\mathbb{R}^{2}$ into rectangles, we obtain a separation condition which satisfies the hypothesis of [10, Theorem 2.3], and then we define a more general function space $X_{p}^{q, r}$ than $X_{p}$ in [12] (see Definition 3.1 below). Since [10, Theorem 2.3] is valid not only for paraboloid cases but also for some hyperbolic cases, a refinement of Strichartz estimates in [12] could be extended to our mixed-norm case. This refinement is especially meaningful in that it enables the decomposition of initial data $u_{0}(x)$ into 
a finite sequence of functions, which will be described precisely in Lemma 3.7. We will also make use of some mixed-norm estimates on the space $X_{p}^{q, r}$ which are adapted from the results in [4].

It is worthwhile to make the following remarks which allow us to restrict the range of an admissible pair $(q, r)$ to $q \leq r \leq 6$.

REMARK 1.2. It suffices to consider only the case $q \leq r$. To see this, observe that if $\|u\|_{L_{t}^{q} L_{x}^{r}\left(\left[0, T_{\max }\right) \times \mathbb{R}^{2}\right)}=\infty$ for $q \geq r$, then $\|u\|_{L_{t, x}^{4}\left(\left[0, T_{\max }\right) \times \mathbb{R}^{2}\right)}=\infty$ from interpolation with the mass conservation $\|u\|_{L_{t}^{\infty} L_{x}^{2}}=\left\|u_{0}\right\|_{L_{x}^{2}\left(\mathbb{R}^{2}\right)}$. Indeed, let $\left(q_{0}, r_{0}\right)$ be an admissible pair with $q_{0} \geq r_{0}$ such that

$$
\frac{1}{q_{0}}=\frac{1-\theta}{\infty}+\frac{\theta}{4} \text { and } \quad \frac{1}{r_{0}}=\frac{1-\theta}{2}+\frac{\theta}{4}
$$

for some $\theta \in(0,1)$. If $\|u\|_{L_{t}^{q_{0}} L_{x}^{r_{0}}}=\infty$, then $\|u\|_{L_{t, x}^{4}}=\infty$ follows from

$$
\|u\|_{L_{t}^{q_{0}} L_{x}^{r_{0}}} \leq\left(\sup _{t}\|u\|_{L_{x}^{2}}\right)^{1-\theta}\|u\|_{L_{t, x}^{4}}^{\theta} \quad \text { and } \quad \sup _{t}\|u\|_{L_{x}^{2}}=\left\|u_{0}\right\|_{L_{x}^{2}} \neq 0
$$

by Hölder's inequality and the conservation of charge. Hence, there exists a mass concentration phenomenon by the result in [12].

REMARK 1.3. For the local well-posedness of (1.1) in the mixed-norm space $L_{t}^{q} L_{x}^{r}$, we would check if the inhomogeneous part of the solution is a contraction map. Actually, by Duhamel's principle, the solution to (1.1) is given by

$$
u(t, x)=e^{i t \square} u_{0}(x)+i \gamma \int_{0}^{t} e^{i(t-s) \square}|u(s)|^{2} u(s) d s .
$$

Using (1.3), the inhomogeneous Strichartz estimate in Lemma 2.1 below and Hölder's inequality, it follows that for any admissible pairs $(q, r)$ and $(\tilde{q}, \tilde{r})$,

$$
\begin{aligned}
& \left\|\int_{0}^{T} e^{i(t-s) \square}\left[|u(s)|^{2} u(s)-|v(s)|^{2} v(s)\right] d s\right\|_{L_{t}^{q} L_{x}^{r}} \\
& \leq|||u|^{2} u-|v|^{2} v \|_{L_{t}^{\tilde{q}^{\prime}} L_{x}^{\tilde{r}^{\prime}}} \\
& =\left\|\left(|u|^{2}-|v|^{2}\right) u+|v|^{2}(u-v)\right\|_{L_{t}^{\tilde{q}^{\prime}} L_{x}^{\tilde{r}^{\prime}}} \\
& =\left\|(|u|-|v|)(|u|+|v|) u+|v|^{2}(u-v)\right\|_{L_{t}^{\tilde{q}^{\prime}} L_{x}^{\tilde{r}^{\prime}}} \\
& \leq|||u-v|\left((|u|+|v|)|u|+|v|^{2}\right) \|_{L_{t}^{\tilde{q}^{\prime}} L_{x}^{\tilde{r}^{\prime}}} \\
& \leq C\left\|\left(|u|^{2}+|v|^{2}\right) \mid u-v\right\| \|_{L_{t}^{\tilde{q}^{\prime}} L_{x}^{\tilde{r}^{\prime}}} \\
& \leq C|||u|^{2}+|v|^{2}\left\|_{L_{t}^{\frac{3}{2} \tilde{q}^{\prime}} L_{x}^{\frac{3}{2} \tilde{r}^{\prime}}}\right\| u-v \|_{L_{t}^{3 \tilde{q}^{\prime}} L_{x}^{3 \tilde{r}^{\prime}}} \\
& \leq C\left(\|u\|_{L_{t}^{3 \tilde{\tilde{q}^{\prime}}} L_{x}^{3 \tilde{r}^{\prime}}}^{2}+\|v\|_{L_{t}^{3 \tilde{\tilde{q}^{\prime}}} L_{x}^{3 \tilde{r}^{\prime}}}^{2}\|u-v\|_{L_{t}^{3 \tilde{q}^{\prime}} L_{x}^{3 r^{\prime}}} .\right.
\end{aligned}
$$

The conditions $q=3 \tilde{q}^{\prime}$ and $r=3 \tilde{r}^{\prime}$ imply that $1 / 6 \leq 1 / r \leq 1 / 3$. For this range of $r,(1.1)$ is locally well-posed in the mixed norm space $C\left([0, T] ; L^{2}\left(\mathbb{R}^{2}\right)\right) \cap L^{q}\left([0, T] ; L^{r}\left(\mathbb{R}^{2}\right)\right)$ for a small time $T<T_{\max }$. 
REMARK 1.4. For observing a mass concentration phenomenon, the inhomogeneous part of the solution does not play a primary role as long as the integral part in (1.3) can be controlled by the solution $u(t, x)$. For example, there may be a mass concentration phenomenon for the hyperbolic-elliptic type Davey-Stewartson system, with subsonic wave packet, which is defined by

$$
i u_{t}-\partial_{x_{1}}^{2} u+\partial_{x_{2}}^{2} u=\left( \pm|u|^{2}+\mathcal{B}\left(|u|^{2}\right)\right) u
$$

where

$$
\widehat{\mathcal{B}(f)}\left(\xi_{1}, \xi_{2}\right)=\frac{-\gamma \xi_{1}^{2}}{\xi_{1}^{2}+\xi_{2}^{2}} \hat{f}\left(\xi_{1}, \xi_{2}\right) \quad \text { and } \quad \gamma>0 .
$$

A detailed discussion of the Davey-Stewartson system may be found in [13].

In practice, it suffices to show that

$$
\left\| \pm|u|^{2} u+\mathcal{B}\left(|u|^{2}\right) u\right\|_{L_{t}^{\tilde{q}} L_{x}^{\tilde{r}}\left(\left(T_{0}, T_{1}\right) \times \mathbb{R}^{2}\right)} \leq C\|u\|_{L_{t}^{q} L_{x}^{r}}^{3}
$$

for $q=3 \tilde{q}$ and $r=3 \tilde{r}$.

Note that $\|\mathcal{B}(f)\|_{L_{x}^{p}} \leq C\|f\|_{L_{x}^{p}}$ for $1<p<\infty$ by the Marcinkiewicz multiplier theorem. Thus

$$
\begin{aligned}
\left\|\mathcal{B}\left(|u|^{2}\right) u\right\|_{L_{t}^{\tilde{q}} L_{x}^{\tilde{x}}} & \leq\left\|\mathcal{B}\left(|u|^{2}\right)\right\|_{L_{t}^{q / 2} L_{x}^{r / 2}}\|u\|_{L_{t}^{q} L_{x}^{r}} \\
& \leq\|C\||u|^{2}\left\|_{L_{x}^{r / 2}}\right\|_{L_{t}^{q / 2}}\|u\|_{L_{t}^{q} L_{x}^{r}} \leq C\|u\|_{L_{t}^{q} L_{x}^{r}}^{3}
\end{aligned}
$$

Using the triangle inequality, we obtain the desired result.

This paper is organized as follows. In Section 2 we obtain some Strichartz estimates for the operator $e^{i t \square}$, which is proved in the same manner as in the case of the Schrödinger operator $e^{i t \Delta}$. In Section 4 we give a proof of Theorem 1.1. In Section 3 we prove some useful and technical lemmas which are used in Section 4.

\section{Strichartz estimates}

In this section a brief review of Strichartz estimates will be given. The following argument may be found in [3, 7] or [15].

To get the dual operator of $e^{i t \square}$, we need the following calculation:

$$
\begin{aligned}
\left\langle e^{i t \square} u_{0}(x), v(t, x)\right\rangle & =\iint_{\mathbb{R}} \int_{\mathbb{R}^{2}} e^{i t \square} u_{0}(x) \overline{v(t, x)} d x d t \\
& =\iiint \int u_{0}(y) e^{-2 \pi i y \cdot \xi} e^{2 \pi i\left(x \cdot \xi-2 \pi t \xi_{1} \xi_{2}\right)} \overline{v(t, x)} d \xi d y d x d t \\
& =\int u_{0}(y) \overline{\iint \hat{v}(t, \xi) e^{2 \pi i\left(y \cdot \xi+2 \pi t \xi_{1} \xi_{2}\right)} d \xi d t} d y .
\end{aligned}
$$


Hence, the dual operator of $e^{i t \square} F(x)$ is $\int e^{-i t \square} F_{t}(x) d t$, where $F=F(t, x)=F_{t}(x)$.

Our claim is that

$$
\left\|\int_{\mathbb{R}} e^{-i t \square} F_{t} d t\right\|_{L_{x}^{2}\left(\mathbb{R}^{2}\right)} \lesssim\|F\|_{L_{t}^{q^{\prime}} L_{x}^{r^{\prime}}\left(\mathbb{R} \times \mathbb{R}^{2}\right)}
$$

for every $F \in L_{t}^{q^{\prime}} L_{x}^{r^{\prime}}\left(\mathbb{R} \times \mathbb{R}^{2}\right)$ and any admissible pair $(q, r)$. Here and throughout this paper, $q^{\prime}$ denotes the conjugate exponent of $q$ defined by $1 / q+1 / q^{\prime}=1$.

Let $(q, r)$ be an admissible pair with $2 \leq q, r \leq \infty$ and $(q, r) \neq(2, \infty)$. Then

$$
\begin{aligned}
\left\|\int_{\mathbb{R}} e^{-i s \square} F_{s} d s\right\|_{L_{x}^{2}}^{2} & =\iint e^{-i s \square} F_{s} d t \overline{\int e^{-i t \square} F_{t} d t} d x \\
& =\iint\left\langle e^{-i s \square} F_{s}, e^{-i t \square} F_{t}\right\rangle d s d t \\
& =\iint\left\langle e^{i(t-s) \square} F_{s}, F_{t}\right\rangle d s d t \\
& =\iiint e^{i(t-s) \square} F_{s} \overline{F_{t}(x)} d x d t d s \\
& =\iiint e^{i(t-s) \square} F_{s} d s \overline{F_{t}(x)} d t d x \\
& \leq \int\left(\int\left|\int e^{i(t-s) \square} F_{s} d s\right|^{q} d t\right)^{1 / q}\left(\int|F|^{q^{\prime}} d t\right)^{1 / q^{\prime}} d x \\
& \leq\left\|\int e^{i(t-s) \square} F_{s} d s\right\|\left\|_{L_{t}^{q} L_{x}^{r}}\right\| F \|_{L_{t}^{q^{\prime}} L_{x}^{r^{\prime}}}
\end{aligned}
$$

Now, by Minkowski’s inequality,

$$
\begin{aligned}
\left\|\int e^{i(t-s) \square} F_{s} d s\right\|_{L_{t}^{q} L_{x}^{r}} & =\left(\int\left(\int\left|\int e^{i(t-s) \square} F_{s} d s\right|^{r} d x\right)^{q / r} d t\right)^{1 / q} \\
& \leq\left\|\int_{\mathbb{R}}\right\| e^{i(t-s) \square} F_{s}\left\|_{L_{x}^{r}\left(\mathbb{R}^{2}\right)} d s\right\|_{L_{t}^{q}(\mathbb{R})} .
\end{aligned}
$$

Let us assume for the moment that $\left\|e^{i t \square} F_{t}\right\|_{L_{x}^{r}\left(\mathbb{R}^{2}\right)} \leq C|t|^{-2(1 / 2-1 / r)}\left\|F_{t}\right\|_{L_{x}^{r^{\prime}\left(\mathbb{R}^{2}\right)}}$ for $2 \leq r \leq \infty$. Whenever $(q, r)$ is an admissible pair with $2<q<\infty$ and $2<r<\infty$, it follows by the Hardy-Littlewood-Sobolev inequality that there is a positive constant $C$ such that

$$
\begin{aligned}
\left\|\int_{\mathbb{R}}\right\| e^{i(t-s) \square} F_{s}\left\|_{L_{x}^{r}\left(\mathbb{R}^{2}\right)} d s\right\|_{L_{t}^{q}(\mathbb{R})} & \leq C\left\|\int_{\mathbb{R}}|t-s|^{-2(1 / 2-1 / r)}\right\| F_{s}\left\|_{L_{x}^{r^{\prime}}\left(\mathbb{R}^{2}\right)} d s\right\|_{L_{t}^{q}(\mathbb{R})} \\
& \leq C\|\| F_{s}\left\|_{L_{x}^{r^{\prime}\left(\mathbb{R}^{2}\right)}}\right\|_{L_{t}^{q^{\prime}(\mathbb{R})}}=C\|F\|_{L_{t}^{q^{\prime}} L_{x}^{r^{\prime}}}
\end{aligned}
$$


We need to show that $\left\|e^{i t \square} F_{t}\right\|_{L_{x}^{r}} \leq(2 \pi|t|)^{-2(1 / 2-1 / r)}\left\|F_{t}\right\|_{L_{x}^{r^{\prime}}}$. We begin with

$$
\begin{aligned}
e^{i t \square} F_{t}(x) & =\int \hat{F}_{t}(\xi) e^{2 \pi i\left(x \cdot \xi-2 \pi t \xi_{1} \xi_{2}\right)} d \xi \\
& =\iint F(t, y) e^{-2 \pi i y \cdot \xi} d y e^{2 \pi i\left(x \cdot \xi-2 \pi t \xi_{1} \xi_{2}\right)} d \xi \\
& =\iint e^{2 \pi i(x-y) \cdot \xi} e^{-4 \pi i t \xi_{1} \xi_{2}} d \xi F(t, y) d t .
\end{aligned}
$$

If we simplify the phase by making a change of variables,

$$
\begin{aligned}
\int e^{2 \pi i x \cdot \xi} e^{-4 \pi i t \xi_{1} \xi_{2}} d \xi & =2 \int e^{2 \pi i x \cdot\left(\zeta_{1}+\zeta_{2}, \zeta_{1}-\zeta_{2}\right)} e^{-4 \pi i t\left(\zeta_{1}^{2}-\zeta_{2}^{2}\right)} d \zeta_{1} d \zeta_{2} \\
& =2 \int e^{2 \pi i\left(x_{1}+x_{2}\right) \zeta_{1}} e^{-4 \pi i t \zeta_{1}^{2}} d \zeta_{1} \int e^{2 \pi i\left(x_{1}-x_{2}\right) \zeta_{2}} e^{-4 \pi i t \zeta_{2}^{2}} d \zeta_{2} \\
& =2\left(\frac{1}{4 \pi i t}\right)^{1 / 2} e^{i\left(x_{1}+x_{2}\right)^{2} / 4 t}\left(\frac{1}{4 \pi i t}\right)^{1 / 2} e^{i\left(x_{1}-x_{2}\right)^{2} /(-4 t)} \\
& =\frac{1}{2 \pi i t} e^{(i / t)\left(x_{1} x_{2}\right)} .
\end{aligned}
$$

Therefore,

$$
e^{i t \square} F_{t}(x)=\frac{1}{2 \pi i t} \int_{\mathbb{R}^{2}} e^{i\left(x_{1}-y_{1}\right)\left(x_{2}-y_{2}\right) / t} F(t, y) d t .
$$

Hence, we get two estimates as follows:

$$
\begin{aligned}
\left\|e^{i t \square} F_{t}\right\|_{L_{x}^{\infty}} & \leq(2 \pi t)^{-1}\left\|F_{t}\right\|_{L_{x}^{1}} \\
\left\|e^{i t \square} F_{t}\right\|_{L_{x}^{2}} & \leq\left\|F_{t}\right\|_{L_{x}^{2}} .
\end{aligned}
$$

By interpolating these two estimates, it follows that for $2 \leq r \leq \infty$,

$$
\left\|e^{i t \square} F_{t}\right\|_{L^{r}\left(\mathbb{R}^{2}\right)} \leq(2 \pi|t|)^{-2(1 / 2-1 / r)}\left\|F_{t}\right\|_{L^{r^{\prime}\left(\mathbb{R}^{2}\right)}} .
$$

Hence we have the following lemma.

Lemma 2.1 (An inhomogeneous Strichartz estimate). Let $(q, r)$ and $(\tilde{q}, \tilde{r})$ be admissible pairs satisfying $2 \leq q, r, \tilde{q}, \tilde{r} \leq \infty,(q, r) \neq \infty$ and $(\tilde{q}, \tilde{r}) \neq \infty$. Then for every $F \in L_{t}^{\tilde{q}^{\prime}} L_{x}^{\tilde{r}^{\prime}}\left(\mathbb{R} \times \mathbb{R}^{2}\right)$ and $u_{0} \in L_{x}^{2}\left(\mathbb{R}^{2}\right)$,

$$
\left\|\int_{\mathbb{R}} e^{-i s \square} F_{s} d s\right\|_{L_{x}^{2}\left(\mathbb{R}^{2}\right)} \lesssim\|F\|_{L_{t}^{\tilde{q}^{\prime}} L_{x}^{\tilde{r}^{\prime}}\left(\mathbb{R} \times \mathbb{R}^{2}\right)} \quad \text { (dual homogeneous) }
$$

and by duality,

$$
\left\|e^{i t \square} u_{0}\right\|_{L_{t}^{q} L_{x}^{r}} \lesssim\left\|u_{0}\right\|_{L_{x}^{2}\left(\mathbb{R}^{2}\right)} \quad \text { (homogeneous). }
$$

Moreover, for $t_{0}<t$,

$$
\left\|\int_{t_{0}}^{t} e^{i(t-s) \square} F_{s} d s\right\|_{L_{t}^{q} L_{x}^{r}} \lesssim\|F\|_{L_{t}^{\tilde{q}^{\prime}} L_{x}^{\tilde{r}^{\prime}}} \quad \text { (inhomogeneous). }
$$

All omitted constants are positive and depend only on $(q, r)$ or $(\tilde{q}, \tilde{r})$. 
In fact,

$$
\left\|\int_{\mathbb{R}} e^{i(t-s) \square} F_{s} d s\right\|_{L_{t}^{q} L_{x}^{r}} \lesssim\|F\|_{L_{t}^{\tilde{q}^{\prime}} L_{x}^{\tilde{r}^{\prime}}}
$$

by (2.1) and (2.2). Then (2.3) follows from the Christ-Kiselev lemma in [6].

\section{Proofs of lemmas}

The purpose of this section is to prove Lemmas 3.7 and 3.9. We begin with a new function space whose definition is adapted from that of $X_{p}$ in $[11,12]$.

Definition 3.1. For each $k, l \in \mathbb{Z}$, we break $\mathbb{R}^{2}$ up into rectangles $R_{k, l}^{j}$ such that

$$
R_{k, l}^{j}=\left[j_{1} 2^{-k},\left(j_{1}+1\right) 2^{-k}\right] \times\left[j_{2} 2^{-l},\left(j_{2}+1\right) 2^{-l}\right]
$$

where $j=\left(j_{1}, j_{2}\right) \in \mathbb{Z}^{2}$. We define a function space $X_{p}^{q, r}$ by

$$
X_{p}^{q, r}=\left\{f:\|f\|_{X_{p}^{q, r}}=\left[\sum_{k, l}\left(\sum_{j}\left(2^{(k+l)(1 / p-1 / 2)}\left(\int_{R_{k, l}^{j}}|f|^{p}\right)^{1 / p}\right)^{r}\right)^{q / r}\right]^{1 / q}<\infty\right\}
$$

for $1 \leq p, q, r \leq \infty$. When an index is $\infty$, we adopt the usual supremum norm interpretation for the corresponding norm.

Then we can observe the following properties of $X_{p}^{q, r}$.

Lemma 3.2. If $p<2<\min \{q, r\}$, then for some $0<\theta<1$, there exists a constant $C$ such that

$$
\|f\|_{X_{p}^{q, r}} \leq C \sup _{j, k, l}\left(\left|R_{k, l}^{j}\right|^{(1 / 2-1 / p)}\left(\int_{R_{k, l}^{j}}|f|^{p}\right)^{1 / p}\right)^{\theta}\|f\|_{L^{2}}^{1-\theta} .
$$

Proof. For $q \leq r$, we have $\|f\|_{X_{p}^{q, r}} \leq\|f\|_{X_{p}^{q, q}}$. Clearly,

$$
\|f\|_{X_{p}^{\infty, \infty}} \leq \sup _{j, k, l} 2^{(k+l)(1 / p-1 / 2)}\left(\int_{R_{k, l}^{j}}|f|^{p}\right)^{1 / p}
$$

If we show that

$$
\|f\|_{X_{p}^{s, s}} \leq C\|f\|_{L^{2}}
$$

for $p<2<s$, then, by interpolation between (3.1) and (3.2),

$$
\|f\|_{X_{p}^{q, r}} \leq\|f\|_{X_{p}^{q, q}} \leq\left(\sup _{j, k, l} 2^{(k+l)(1 / p-1 / 2)}\left(\int_{R_{k, l}^{j}}|f|^{p}\right)^{1 / p}\right)^{1-s / q}\|f\|_{L^{2}}^{s / q}
$$

as long as we choose $s$ smaller than $q$.

To prove (3.2), we may assume that $\|f\|_{L^{2}}=1$. We decompose $f$ into $f^{m}$ and $f_{m}$ where $f^{m}=f \chi_{\left\{|f| \geq 2^{(k+l) / 2}\right\}}$ and $f_{m}=f \chi_{\left\{|f|<2^{(k+l) / 2}\right\}}$, respectively. 
First, for $p<2<s$, there is a constant $C_{1}=C_{1}(p)$ such that

$$
\begin{aligned}
\sum_{j} \sum_{k, l}\left(2^{(k+l)(1 / p-1 / 2)}\left(\int_{R_{k, l}^{j}}\left|f^{m}\right|^{p}\right)^{1 / p}\right)^{s} & \leq\left(\sum_{j} \sum_{k, l} 2^{(k+l)(1 / p-1 / 2) p} \int_{R_{k, l}^{j}}\left|f^{m}\right|^{p}\right)^{s / p} \\
& =\left(\int \sum_{k, l} 2^{(k+l)(1 / p-1 / 2) p}\left|f^{m}\right|^{p}\right)^{s / p} \\
& =\left(\int \sum_{|f| \geq 2^{(k+l) / 2}} 2^{(k+l)(1 / p-1 / 2) p}|f|^{p}\right)^{s / p} \\
& \leq C_{1}\left(\int|f|^{2 p(1 / p-1 / 2)+p}\right)^{s / p}=C_{1}\|f\|_{L^{2}}^{2 s / p} \leq C_{1}
\end{aligned}
$$

Using Hölder's inequality, we also know that there is a constant $C_{2}=C_{2}(s)$ such that

$$
\begin{aligned}
\sum_{j} \sum_{k, l}\left(2^{(k+l)(1 / p-1 / 2)}\left(\int_{R_{k, l}^{j}}\left|f_{m}\right|^{p}\right)^{1 / p}\right)^{s} & \leq \sum_{j} \sum_{k, l} 2^{(k+l)(1 / s-1 / 2) s} \int_{R_{k, l}^{j}}\left|f^{m}\right|^{s} \\
& =\int_{\mathbb{R}^{2}} \sum_{k, l} 2^{(k+l)(1 / s-1 / 2) s}\left|f_{m}\right|^{s} \\
& =\int_{\mathbb{R}^{2}} \sum_{|f|<2^{(k+l) / 2}} 2^{(k+l)(1 / s-1 / 2) s}|f|^{s} \\
& \leq C_{2} \int_{\mathbb{R}^{2}}|f|^{2 s(1 / s-1 / 2)+s}=C_{2}\|f\|_{L^{2}}^{2}=C_{2} .
\end{aligned}
$$

As a result, we can choose a constant $C=C(p, s)$ satisfying $\|f\|_{X_{p}^{s, s}} \leq C\|f\|_{L^{2}}$ when $p<2<s$.

On the other hand, for the case $r \leq q$, we have $X_{p}^{r, r} \subset X_{p}^{q, r}$ and so we obtain again the estimate (3.2) for any $2<s<r$. This completes the proof.

To prove Lemma 3.5 stated below, we need some results about bilinear extension estimates on the saddle surface. Fortunately, [10, Theorem 2.3], which is a sort of mixed-norm generalization of the results in $[8,14,17]$, is useful in our case. The following theorem is taken from [10].

Theorem 3.3 [10]. Assume that $n \geq 2$. Let $\phi_{1}$ and $\phi_{2}$ be smooth functions defined on $[-1,1]^{n-1}$. Define an operator $E_{i} f(x, t)$, for $i=1,2$, by

$$
E_{i} f(x, t)=\int_{[-1,1]^{n-1}} e^{i\left(x \cdot \xi+t \phi_{i}(\xi)\right)} f(\xi) d \xi
$$


Also, denote the Hessian matrix of $\phi$ by $H \phi$. If $\operatorname{det} H \phi_{i} \neq 0$ on $[-1,1]^{n-1}$ and for all $\xi, \zeta \in[-1,1]^{n-1}$,

$$
\left|\left\langle H \phi_{i}^{-1}\left(\nabla \phi_{1}(\xi)-\nabla \phi_{2}(\zeta)\right), \nabla \phi_{1}(\xi)-\nabla \phi_{2}(\zeta)\right\rangle\right| \geq c>0,
$$

then for $2<q, r$ satisfying $2 / q<\min (1, n / 4)$ and $4 / q<n(1-2 / r)$, there is $a$ constant $C$ such that

$$
\left\|E_{1}\left(f_{1}\right) E_{2}\left(f_{2}\right)\right\|_{L_{t}^{q / 2} L_{x}^{r / 2}} \leq C\left\|f_{1}\right\|_{L^{2}}\left\|f_{2}\right\|_{L^{2}}
$$

Remark 3.4. From [9, Theorem 5.1], the condition $4 / q<n(1-2 / r)$ could be extended to $2 / q<2-1 / r$ when $n=3$. Then the range of $p$ in (3.5) or (3.6) is also extended. In particular, we may substitute $\frac{16}{13}$ for the infimum $\frac{12}{7}$ of $p^{*}$ in Lemma 3.5. Nevertheless, the conditions in Theorem 3.3 are enough to prove Lemma 3.5.

Note that the line segment $1 / q=1 / 2-1 / r$ with $3<r \leq 6$ is contained in the area given by $1 / q<3 / 8$ and $1 / q<\frac{3}{4}(1-2 / r)$. By interpolation between (3.4) and a trivial $L^{1}-L^{\infty}$ estimate, we can conclude that there is a constant $C$ such that

$$
\left\|E_{1}\left(f_{1}\right) E_{2}\left(f_{2}\right)\right\|_{L_{t}^{q_{0} / 2} L_{x}^{r_{0} / 2}} \leq C\left\|f_{1}\right\|_{L^{p}}\left\|f_{2}\right\|_{L^{p}}
$$

for some $1<p<2$ determined by a given $\left(q_{0}, r_{0}\right)$ pair satisfying $1 / q_{0}=1 / 2-1 / r_{0}$ and $3<r_{0} \leq 6$ (see Figure 1). More precisely, $1 / p=1-\theta / 2$ with $\theta=q / q_{0}=r / r_{0}$.

In our case, if $R_{k, l}^{j}$ and $R_{k, l}^{j^{\prime}}$ are separated such that $\xi \in R_{k, l}^{j}$ and $\zeta \in R_{k, l}^{j^{\prime}}$ satisfy (3.3), a simple change of variables gives us

$$
\left\|e^{i t \square} f_{k, l}^{j} e^{i t \square} f_{k, l}^{j^{\prime}}\right\|_{L_{t}^{q / 2} L_{x}^{r / 2}} \leq C 2^{(k+l)(2 / r+2 / q-2+2 / p)}\left\|\hat{f}_{k, l}^{j}\right\|_{L^{p}}\left\|\hat{f}_{k, l}^{j^{\prime}}\right\|_{L^{p}}
$$

where $f_{k, l}^{j}$ is the inverse Fourier transform of $\hat{f}_{k, l}^{j}=\hat{f} \chi_{R_{k, l}^{j}}$ supported in a rectangle $R_{k, l}^{j}$. LEMMA 3.5. Let $(q, r)$ be an admissible pair with $2<q \leq 4 \leq r$. Then there is a constant $C=C(q, r)$ such that

$$
\left\|e^{i t \square} f\right\|_{L_{t}^{q} L_{x}^{r}} \leq\|\hat{f}\|_{X_{p^{*}}^{q, q}}
$$

for some $p^{*}$ with $12 / 7<p^{*}<2$.

Proof. We use the notation and terminology in [12] to decompose $\mathbb{R}^{2}$. Let $R_{k, l}^{j}$ be a rectangle of dimension $2^{-k} \times 2^{-l}$ as in Definition 3.1. We consider the rectangles $R_{k-1, l-1}^{j_{1}}, R_{k-1, l}^{j_{2}}$ and $R_{k, l-1}^{j_{3}}$ containing $R_{k, l}^{j}$ as the mother, father, and stepfather, respectively. If $R_{k, l}^{j}$ and $R_{k, l}^{j^{\prime}}$ have adjacent mothers, but their fathers and stepfathers are not adjacent, we use the notation $R_{k, l}^{j} \sim R_{k, l}^{j^{\prime}}$ or simply $j \sim j^{\prime}$. Then

$$
\left\|e^{i t \square} f\right\|_{L_{t}^{q} L_{x}^{r}}^{2}=\left\|e^{i t \square} f e^{i t \square} f\right\|_{L_{t}^{q / 2} L_{x}^{r / 2}}=\|\| \sum_{k, l} \sum_{j \sim j^{\prime}} e^{i t \square} f_{k, l}^{j} e^{i t \square} f_{k, l}^{j^{\prime}}\left\|_{L_{x}^{r / 2}}\right\|_{L_{t}^{q / 2}} .
$$




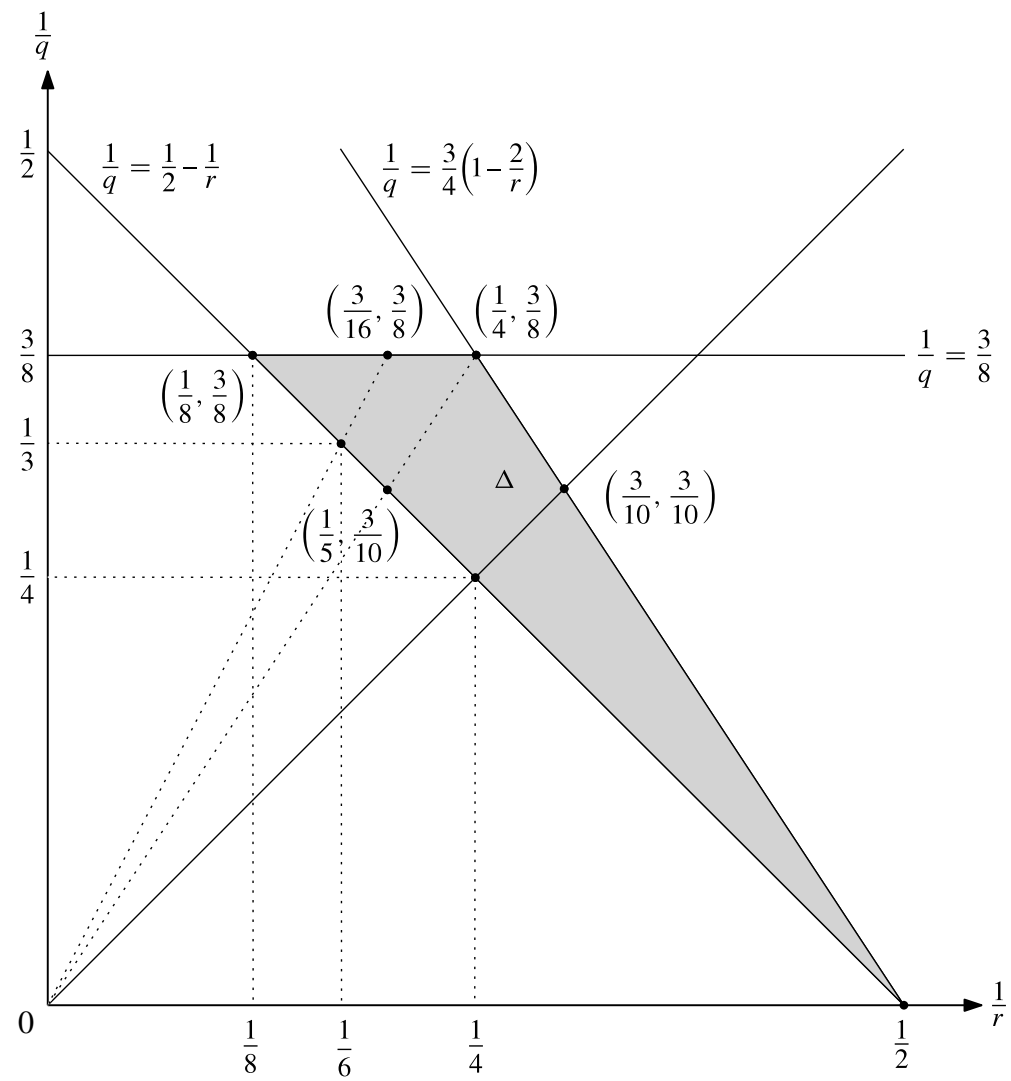

FIGURE 1 . For any given admissible pair $\left(q_{0}, r_{0}\right)$ with $1 / q_{0}<3 / 8$, there is a pair $\left(q_{1}, r_{1}\right) \in \Delta$. So we can determine $p$ in (3.5) using the ratio of $q_{0}$ to $q_{1}$.

Now let us assume for the moment that

$$
\left\|\sum_{k, l} \sum_{j \sim j^{\prime}} e^{i t \square} f_{k, l}^{j} e^{i t \square} f_{k, l}^{j^{\prime}}\right\|_{L_{x}^{r / 2}} \leq C\left(\sum_{k, l} \sum_{j \sim j^{\prime}}\left\|e^{i t \square} f_{k, l}^{j} e^{i t \square} f_{k, l}^{j^{\prime}}\right\|_{L_{x}^{r / 2}}^{q / 2}\right)^{2 / q} .
$$

Then, using the fact that $(q, r)$ is an admissible pair, together with (3.6) and the Cauchy-Schwarz inequality, we can say that

$$
\begin{aligned}
\left\|\sum_{k, l} \sum_{j \sim j^{\prime}} e^{i t \square} f_{k, l}^{j} e^{i t \square} f_{k, l}^{j^{\prime}}\right\|_{L_{t}^{q / 2} L_{x}^{r / 2}} & \leq C\left\|\left(\sum_{k, l} \sum_{j \sim j^{\prime}}\left\|e^{i t \square} f_{k, l}^{j} e^{i t \square} f_{k, l}^{j^{\prime}}\right\|_{L_{x}^{r / 2}}^{q / 2}\right)^{2 / q}\right\|_{L_{t}^{q / 2}} \\
& \leq C\left(\sum_{k, l} \sum_{j \sim j^{\prime}}\left\|e^{i t \square} f_{k, l}^{j} e^{i t \square} f_{k, l}^{j^{\prime}}\right\|_{L_{t}^{q / 2} L_{x}^{r / 2}}^{q / 2}\right)^{2 / q} \\
& \leq C\left(\sum_{k, l} \sum_{j \sim j^{\prime}}\left(2^{(k+l)\left(2 / p^{*}-1\right)}\left\|\hat{f}_{k, l}^{j}\right\|_{L^{p^{*}}}\left\|\hat{f}_{k, l}^{j^{\prime}}\right\|_{L^{p^{*}}}\right)^{q / 2}\right)^{2 / q}
\end{aligned}
$$




$$
\begin{aligned}
& \leq C\left(\sum_{k, l} 2^{(k+l)\left(2 / p^{*}-1\right)(q / 2)} \sum_{j}\left\|\hat{f}_{k, l}^{j}\right\|_{L^{p^{*}}}^{q}\right)^{2 / q} \\
& =C\left(\sum_{k, l} \sum_{j}\left(2^{(k+l)\left(1 / p^{*}-1 / 2\right)}\left\|\hat{f}_{k, l}^{j}\right\|_{L^{p^{*}}}\right)^{q}\right)^{2 / q}
\end{aligned}
$$

for some $12 / 7<p^{*}<2$ determined by an admissible pair $(q, r)$ by $(3.5)$.

Thus,

$$
\left\|e^{i t \square} f\right\|_{L_{t}^{q} L_{x}^{r}} \leq C\left(\sum_{k, l} \sum_{j}\left(2^{(k+l)\left(1 / p^{*}-1 / 2\right)}\left\|\hat{f}_{k, l}^{j}\right\|_{L^{p^{*}}}\right)^{q}\right)^{1 / q}=C\|\hat{f}\|_{X_{p^{*}}^{q, q}}
$$

We now turn to the proof of (3.7) for an admissible pair $(q, r)$. For each $t$, the support of the Fourier transform of $e^{i t \square} \hat{f}_{k, l}^{j} e^{i t \square} \hat{f}_{k, l}^{j^{\prime}}$ in $x$ is contained in $R_{k, l}^{j}+R_{k, l}^{j^{\prime}}$, which is a subset of $\tilde{R}_{k, l}^{j}=\left\{\left(m_{1}, m_{2}\right) \in \mathbb{R}^{2}:\left|m_{1}-\left(j_{1}+3\right) 2^{-k+1}\right| \leq C 2^{-k},\left|m_{2}-\left(j_{2}+3\right) 2^{-l+1}\right| \leq C 2^{-l}\right\}$. It is easy to verify that $\sum_{k, l} \sum_{j \sim j^{\prime}} \chi_{\tilde{R}_{k, l}^{j}}$ is bounded and also that $2 \tilde{R}_{k, l}^{j}$ are almost disjoint. Let us denote by $2 R$ the rectangle with the same center as $R$ and side lengths twice those of $R$. Since $(q, r)$ is admissible and $q \leq r$, we have $q / 2=(r / 2)^{\prime}=\min \left(r / 2,(r / 2)^{\prime}\right)$. Therefore our claim will follow from the following estimate.

Lemma 3.6 [16, Lemma 6.1]. Let $R_{k}$ be a collection of rectangles in frequency space such that the dilates $2 R_{k}$ are almost disjoint, and suppose that $f_{k}$ are a collection of functions whose Fourier transforms are supported on $R_{k}$. Then for all $1 \leq p \leq \infty$,

$$
\left\|\sum_{k} f_{k}\right\|_{p} \lesssim\left(\sum_{k}\left\|f_{k}\right\|_{p}^{p^{*}}\right)^{1 / p^{*}}
$$

where $p^{*}=\min \left(p, p^{\prime}\right)$.

We are now ready to prove the decomposition lemma for the initial datum.

Lemma 3.7. Suppose that $f \in L^{2}\left(\mathbb{R}^{2}\right), 0<\varepsilon \leq\left\|e^{i t \square} f\right\|_{L_{t}^{q} L_{x}^{r}}$ and $(q, r)$ is an admissible pair. Then there exist a natural number $N=N\left(\|f\|_{L^{2}}, \varepsilon\right)$ and a finite sequence of functions $\left\{f_{n}\right\}_{1 \leq n \leq N}$ such that $\hat{f}_{n}$ is supported in a rectangle $R_{n},\left|\hat{f}_{n}\right| \leq A\left|R_{n}\right|^{-1 / 2}$ for some constant $A$, and

$$
\left\|e^{i t \square} f-\sum_{n=1}^{N} e^{i t \square} f_{n}\right\|_{L_{t}^{q} L_{x}^{r}\left(\mathbb{R}^{3}\right)}<\varepsilon .
$$

Proof. By Lemmas 3.2 and 3.5, there exist $p<2$ and a rectangle $R_{1}$ such that

$$
\varepsilon \leq\left\|e^{i t \square} f\right\|_{L_{t}^{q} L_{x}^{r}} \leq C\left(\left|R_{1}\right|^{p / 2-1} \int_{R_{1}}|\hat{f}|^{p}\right)^{(1 / p)(1-\theta)}\|f\|_{L^{2}}^{\theta}
$$

for some $\theta \in(0,1)$. 
It follows that

$$
\int_{R_{1}}|\hat{f}|^{p} \geq\left(\varepsilon\|f\|_{L^{2}}^{-\theta}\right)^{p /(1-\theta)}\left|R_{1}\right|^{1-p / 2}=: c\left|R_{1}\right|^{1-p / 2}
$$

Let $\lambda=\left(2 c^{-1}\|f\|_{L^{2}}^{2}\right)^{1 /(2-p)}\left|R_{1}\right|^{-1 / 2}$. By Plancherel's theorem,

$$
\int_{R_{1} \cap\{|\hat{f}|>\lambda\}}|\hat{f}|^{p}=\int_{R_{1} \cap\{|\hat{f}|>\lambda\}}|\hat{f}|^{p-2}|\hat{f}|^{2} \leq \lambda^{p-2}\|f\|_{L^{2}}^{2} .
$$

On the other hand,

$$
\int_{R_{1} \cap\{|\hat{f}| \leq \lambda\}}|\hat{f}|^{p}=\int_{R_{1}}|\hat{f}|^{p}-\int_{R_{1} \cap\{|\hat{f}|>\lambda\}}|\hat{f}|^{p} \geq c\left|R_{1}\right|^{1-p / 2}-\lambda^{p-2}\|f\|_{L^{2}}^{2} .
$$

By Hölder's inequality,

$$
\int_{R_{1} \cap\{|\hat{f}| \leq \lambda\}}|\hat{f}|^{p} \leq\left(\int_{R_{1} \cap\{|\hat{f}| \leq \lambda\}}|\hat{f}|^{2}\right)^{p / 2}\left|R_{1}\right|^{1-p / 2}
$$

and hence, by (3.8),

$$
\left(\frac{c}{2}\right)^{2 / p} \leq \int_{R_{1} \cap\{|\hat{f}| \leq \lambda\}}|\hat{f}|^{2}
$$

Define $f_{1}$ and $f^{1}$ by $\hat{f}_{1}=\hat{f} \chi_{R_{1} \cap\{|\hat{f}| \leq \lambda\}}$ and $\hat{f}^{1}=\hat{f}-\hat{f}_{1}$. Then $\hat{f}_{1}$ is supported in $\left|R_{1}\right|$ and $\left|\hat{f}_{1}\right| \leq \lambda=A\left|R_{1}\right|^{-1 / 2}$, where

$A=\left(2 c^{-1}\|f\|_{L^{2}}^{2}\right)^{1 /(2-p)}=\left(2\left(\varepsilon\|f\|_{L^{2}}^{-\theta}\right)^{-p /(1-\theta)}\|f\|_{L^{2}}^{2}\right)^{1 /(2-p)}=\left(2 \varepsilon^{-p /(1-\theta)}\|f\|_{L^{2}}^{2+\theta p /(1-\theta)}\right)^{1 /(2-p)}$.

If $\left\|e^{i t \square} f^{1}\right\|_{L_{t}^{q} L_{x}^{r}} \geq \varepsilon$, we repeat the above procedure with $f^{1}$, a rectangle $R_{2}$ and $\lambda_{1}=\left(2 c^{-1}|| f^{1} \|_{L^{2}}^{2}\right)^{1 /(2-p)}\left|R_{2}\right|^{-1 / 2}$ in place of $f, R_{1}$ and $\lambda$. Continuing in this way we get a sequence of functions $\hat{f}_{k-1}=\hat{f}_{k}+\hat{f}^{k}$ where $\hat{f}_{k}$ is supported in a rectangle $R_{k}$, and $\left|\hat{f}_{k}\right| \leq\left(2 c^{-1}\left\|\hat{f}^{k-1}\right\|_{L^{2}}^{2}\right)^{1 /(2-p)}\left|R_{k}\right|^{-1 / 2} \leq\left(2 \varepsilon^{-p /(1-\theta)}\|f\|_{L^{2}}^{2+\theta p /(1-\theta)}\right)^{1 /(2-p)}\left|R_{k}\right|^{-1 / 2}=A\left|R_{k}\right|^{-1 / 2}$.

Furthermore,

$$
\int\left|\hat{f}_{k}\right|^{2} \geq\left(\frac{\left(\varepsilon\left\|\hat{f}^{k-1}\right\|_{L^{2}}^{-\theta}\right)^{p /(1-\theta)}}{2}\right)^{2 / p} \geq\left(\frac{\left(\varepsilon\|f\|_{L^{2}}^{-\theta}\right)^{p /(1-\theta)}}{2}\right)^{2 / p}=\left(\frac{c}{2}\right)^{2 / p} .
$$

Since the $R_{k}$ are pairwise disjoint by construction,

$$
\|\hat{f}\|_{L^{2}}^{2}=\left\|\hat{f}_{1}\right\|_{L^{2}}^{2}+\left\|\hat{f}-\hat{f}_{1}\right\|_{L^{2}}^{2}=\left\|\hat{f}_{1}\right\|_{L^{2}}^{2}+\left\|\hat{f}_{2}\right\|_{L^{2}}^{2}+\left\|\hat{f}-\hat{f}_{1}-\hat{f}_{2}\right\|_{L^{2}}^{2}
$$

and

$$
\left\|\hat{f}-\sum_{j=1}^{n} \hat{f}_{j}\right\|_{L^{2}}^{2}=\|\hat{f}\|_{L^{2}}^{2}-\sum_{j=1}^{n}\left\|\hat{f}_{j}\right\|_{L^{2}}^{2} \leq\|\hat{f}\|_{L^{2}}^{2}-n\left(\frac{c}{2}\right)^{2 / p}
$$


So the Strichartz estimate in Lemma 2.1 and (3.9) imply that

$$
\left\|e^{i t \square} f-\sum_{i=1}^{n} e^{i t \square} f_{j}\right\|_{L_{t}^{q} L_{x}^{r}}^{2} \leq\left\|\hat{f}-\sum_{j=1}^{n} \hat{f}_{j}\right\|_{L^{2}}^{2} \leq\|\hat{f}\|_{L^{2}}^{2}-n\left(\frac{c}{2}\right)^{2 / p} .
$$

As a result, there exists a number $N$ such that

$$
\left\|e^{i t \square} f-\sum_{i=1}^{N} e^{i t \square} f_{j}\right\|_{L_{t}^{q} L_{x}^{r}}<\varepsilon .
$$

The next observation will be useful for proving Lemma 3.9.

Lemma 3.8. Let $2<q \leq r \leq \infty$. Suppose that $\hat{f}$ is supported in the unit square. For any $(q, r)$ satisfying $2 / q+3 / r<3 / 2,2 / q+2 / r<2-2 / q$ and $2 / q>2(1 / 2-1 / r)$, there exists a constant $C=C(q, r)$ such that

$$
\left\|e^{i t \square} f\right\|_{L_{t}^{q} L_{x}^{r}} \leq C\|\hat{f}\|_{L^{\infty}} .
$$

Proof. We may assume that $\|\hat{f}\|_{L^{\infty}}=1$. It suffices to show that $\left\|e^{i t \square} f\right\|_{L_{t}^{q} L_{x}^{r}} \leq C$ for some constant $C$. Let $r^{*}=\min \left(r / 2,(r / 2)^{\prime}\right)$. Then

$$
\begin{aligned}
\left\|\sum_{j \sim j^{\prime}} e^{i t \square} f_{k, l}^{j} e^{i t \square} f_{k, l}^{j^{\prime}}\right\|_{L_{t}^{q / 2} L_{x}^{r / 2}} & \leq \tilde{C}\left\|\left(\sum_{j \sim j^{\prime}}\left\|e^{i t \square} f_{k, l}^{j} e^{i t \square} f_{k, l}^{j^{\prime}}\right\|_{L_{x}^{r / 2}}^{r^{*}}\right)^{1 / r^{*}}\right\|_{L_{t}^{q / 2}} \\
& \leq \tilde{C}\left(\sum_{j \sim j^{\prime}}\left\|e^{i t \square} f_{k, l}^{j} e^{i t \square} f_{k, l}^{j^{\prime}}\right\|_{L_{t}^{q / 2}}^{q / 2} L_{x}^{r / 2}\right)^{2 / q} \\
& \leq \tilde{C}\left(\sum_{j \sim j^{\prime}}\left(2^{(k+l)(2 / r+2 / q-1)}\left\|\hat{f}_{k, l}^{j}\right\|_{L^{2}}\left\|\hat{f}_{k, l}^{j^{\prime}}\right\|_{L^{2}}\right)^{q / 2}\right)^{2 / q} \\
& =\tilde{C} 2^{(k+l)(2 / r+2 / q-1)}\left(\sum_{j \sim j^{\prime}}\left\|\hat{f}_{k, l}^{j}\right\|_{L^{2}}^{q / 2}\left\|\hat{f}_{k, l}^{j^{\prime}}\right\|_{L^{2}}^{q / 2}\right)^{2 / q} \\
& \leq \tilde{C} 2^{(k+l)(2 / r+2 / q-1)}\left(\sum_{j}\left\|\hat{f}_{k, l}^{j}\right\|_{L^{2}}^{q}\right)^{2 / q} .
\end{aligned}
$$

It then follows that

$$
\begin{aligned}
\left\|e^{i t \square} f\right\|_{L_{t}^{q} L_{x}^{r}}^{2} \leq \sum_{k, l}\left\|\sum_{j \sim j^{\prime}} e^{i t \square} f_{k, l}^{j} e^{i t \square} f_{k, l}^{j^{\prime}}\right\|_{L_{t}^{q / 2} L_{x}^{r / 2}} \\
=\tilde{C} \sum_{k+l \geq 0} 2^{(k+l)(2 / r+2 / q-1)}\left(\sum_{j}\left\|\hat{f}_{k, l}^{j}\right\|_{L^{2}}^{q}\right)^{2 / q} \\
\quad+\tilde{C} \sum_{k+l<0} 2^{(k+l)(2 / r+2 / q-1)}\left(\sum_{j}\left\|\hat{f}_{k, l}^{j}\right\|_{L^{2}}^{q}\right)^{2 / q} \\
=: I+I I .
\end{aligned}
$$


First, by Hölder's inequality,

$$
\left(\sum_{j}\left\|\hat{f}_{k, l}^{j}\right\|_{L^{2}}^{q}\right)^{2 / q} \leq\left(\sum_{j}\left\|\hat{f}_{k, l}^{j}\right\|_{L^{q}}^{q}\right)^{2 / q} 2^{(k+l)(2 / q-1)} \leq 2^{(k+l)(2 / q-1)}\|\hat{f}\|_{L^{q}}^{2} .
$$

Hence,

$$
I \leq \tilde{C} \sum_{k+l \geq 0} 2^{(k+l)(4 / q+2 / r-2)}\|\hat{f}\|_{L^{q}}^{2} \leq C_{1}\|\hat{f}\|_{L^{q}}^{2} \leq C_{1} .
$$

The last inequality follows from the fact that $\hat{f}$ is supported in the unit square and $\|\hat{f}\|_{L^{\infty}}=1$.

On the other hand, since $2<q$ and $2 / r+2 / q-1>0$,

$$
I I \leq \tilde{C} \sum_{k+l<0} 2^{(k+l)(2 / r+2 / q-1)} \sum_{j}\left\|\hat{f}_{k, l}^{j}\right\|_{L^{2}}^{2}=\tilde{C} \sum_{k+l<0} 2^{(k+l)(2 / r+2 / q-1)}\|\hat{f}\|_{L^{2}}^{2} \leq C_{2} .
$$

Combining these two estimates, we can conclude that there exists a constant $C=C(q, r)$ such that $\left\|e^{i t \square} f\right\|_{L_{t}^{q} L_{x}^{r}} \leq C$.

From the following lemma, we could find the mass concentrating region.

Lemma 3.9. Let $(q, r)$ be an admissible pair. Suppose that $f \in L^{2}\left(\mathbb{R}^{2}\right)$ and its Fourier transform $\hat{f}$ is supported in a rectangle $R$ with center $\zeta=\left(\zeta_{1}, \zeta_{2}\right)$ and also that $|\hat{f}| \leq A|R|^{-1 / 2}$ for some constant $A>0$. Let $\varepsilon>0$ be given. Then there exists a finite sequence of sets $\left\{Q_{n}\right\}_{1 \leq n \leq N\left(A,\|f\|_{L^{2}}, \varepsilon\right)}$ defined by $Q_{n}=\left\{(t, x) \in \mathbb{R} \times \mathbb{R}^{2}:\left(x_{1}-2 \pi t \zeta_{2}, x_{2}-\right.\right.$ $\left.\left.2 \pi t \zeta_{1}\right) \in R_{n}, t \in I_{n}\right\}$, where $R_{n}$ is a rectangle of measure $|R|^{-1}$ and $I_{n}$ is an interval of length $|R|^{-1}$, such that

$$
\left\|e^{i t \square} f\right\|_{L_{t}^{q} L_{x}^{r}\left(\mathbb{R}^{3} \backslash \cup Q_{n}\right)}<\varepsilon .
$$

Proof. Suppose that a rectangle $R$ has dimensions $2 a \times 2 b$ and center $\zeta$. Then $|\hat{f}| \leq A(a b)^{-1 / 2}$. Now, we make use of a change of variables after a translation $\xi \mapsto \xi+\zeta$ to get a function supported in the unit square:

$$
\begin{aligned}
\left|e^{i t \square} f(x)\right| & =\left|\int \hat{f}(\xi) e^{2 \pi i\left(x \cdot \xi-2 \pi t \xi_{1} \xi_{2}\right)} d \xi\right| \\
& =\left|\int_{\left|\xi_{1}\right| \leq a,\left|\xi_{2}\right| \leq b} \hat{f}(\xi+\zeta) e^{2 \pi i\left(x \cdot(\xi+\zeta)-2 \pi t\left(\xi_{1}+\zeta_{1}\right)\left(\xi_{2}+\zeta_{2}\right)\right)} d \xi\right| \\
& =\left|\int_{\left|\xi_{1}\right| \leq a,\left|\xi_{2}\right| \leq b} \hat{f}(\xi+\zeta) e^{2 \pi i\left(\left(x_{1}-2 \pi t \zeta_{2}, x_{2}-2 \pi t \zeta_{1}\right) \cdot\left(\xi_{1}, \xi_{2}\right)-2 \pi t \xi_{1} \xi_{2}\right)} d \xi\right| \\
& =\mid \int_{\left|\bar{\xi}_{1}\right| \leq 1,\left|\bar{\xi}_{2}\right| \leq 1} \hat{f}\left(\left(a \bar{\xi}_{1}, b \overline{\xi_{2}}\right)+\zeta\right) \\
& \times e^{2 \pi i\left(\left(x_{1}-2 \pi t \zeta_{2}, x_{2}-2 \pi t \zeta_{1}\right) \cdot\left(a \bar{\xi}_{1}, b \bar{\xi}_{2}\right)-2 \pi t a b \bar{\xi}_{1} \bar{\xi}_{2}\right)}(a b) d \bar{\xi}_{1} d \bar{\xi}_{2} \mid \\
= & (a b)^{1 / 2}\left|e^{i t^{\prime} \square} f^{\prime}\left(x^{\prime}\right)\right|
\end{aligned}
$$


where

$$
\hat{f}^{\prime}\left(\xi_{1}, \xi_{2}\right)=(a b)^{1 / 2} \hat{f}\left(\left(a \xi_{1}, b \xi_{2}\right)+\zeta\right), \quad t^{\prime}=a b t
$$

and

$$
x^{\prime}=\left(a\left(x_{1}-2 \pi \zeta_{2}\right), b\left(x_{2}-2 \pi t \zeta_{1}\right)\right) .
$$

Note that $\hat{f}^{\prime}$ is supported in the unit square and that $\left|\hat{f}^{\prime}\right| \leq(a b)^{1 / 2}|\hat{f}| \leq A$. By Lemma 3.8, for any admissible pair $(q, r)$, we can find a pair $(\bar{q}, \bar{r})$ such that $\bar{q}<q$, $\bar{r}<r$ and $\bar{r} / \bar{q}=r / q$, and there is a constant $C$ such that $\left\|e^{i t^{\prime} \square} f^{\prime}\right\|_{L_{t^{\prime}}}^{\bar{q}} L_{x^{\prime}} \leq C\left\|\hat{f}^{\prime}\right\|_{L^{\infty}}$.

Let $E \subset \mathbb{R} \times \mathbb{R}^{2}$ be the set defined by $\left\{\left(t^{\prime}, x^{\prime}\right) \in \mathbb{R} \times \mathbb{R}^{2}:\left|e^{i t^{\prime} \square} f^{\prime}\left(x^{\prime}\right)\right|<\lambda\right\}$ for some $\lambda$. Then

$$
\begin{aligned}
\left\|e^{i t^{\prime} \square} f^{\prime}\right\|_{L_{t^{\prime}}^{q} L_{x^{\prime}}^{r}(E)}^{q} & =\int_{\mathbb{R}}\left(\int_{E_{t^{\prime}}}\left|e^{i t^{\prime} \square} f^{\prime}\left(x^{\prime}\right)\right|^{\bar{r}+r-\bar{r}} d x^{\prime}\right)^{q / r} d t^{\prime} \\
& \leq \lambda^{(r-\bar{r}) q / r}\left\|e^{i t^{\prime} \square} f^{\prime}\right\|_{L_{t^{\prime}}^{\bar{q}} L_{x^{\prime}}^{\bar{r}}}^{\bar{q}} \leq C \lambda^{(r-\bar{r}) q / r}\left\|\hat{f}^{\prime}\right\|_{L^{\infty}}^{\bar{q}} \leq C \lambda^{(r-\bar{r}) q / r} A^{\bar{q}},
\end{aligned}
$$

where $E_{t}=\left\{x \in \mathbb{R}^{2}:(t, x) \in E\right\}$. For a given $\varepsilon$, if we choose

$$
\lambda_{0} \leq \min \left\{2^{-1}\left(C^{-1} A^{-\bar{q}} \varepsilon^{q^{2}}\right)^{r / q(r-\bar{r})}, \frac{1}{4} A\right\}
$$

sufficiently small, we have $\left\|e^{i t^{\prime} \square} f^{\prime}\right\|_{L_{t^{\prime}}^{q} L_{x^{\prime}}^{r}(\tilde{E})} \leq \varepsilon^{q}$ where $\tilde{E}=\left\{\left(t^{\prime}, x^{\prime}\right):\left|e^{i t^{\prime} \square} f^{\prime}\left(x^{\prime}\right)\right|<2 \lambda_{0}\right\}$.

Since $\hat{f}^{\prime}$ is supported in the unit square and $\left|\hat{f}^{\prime}\right| \leq A$, it follows that $\mid e^{i t^{\prime} \square} f^{\prime}\left(x^{\prime}\right)-$ $e^{i t^{\prime \prime} \square} f^{\prime}\left(x^{\prime \prime}\right) \mid \leq c A\left(\left|x^{\prime}-x^{\prime \prime}\right|+\left|t^{\prime}-t^{\prime \prime}\right|\right)$ for some constant $c>1$. If $\left|x^{\prime}-x^{\prime \prime}\right| \leq \lambda_{0} / 2 c A$ and $\left|t^{\prime}-t^{\prime \prime}\right| \leq \lambda_{0} / 2 c A$, then $\left|e^{i t^{\prime} \square} f^{\prime}\left(x^{\prime}\right)\right|<\lambda_{0}$ implies that $\left|e^{i t^{\prime \prime} \square} f^{\prime}\left(x^{\prime \prime}\right)\right|<2 \lambda_{0}$.

So, for some index set $S$, we can choose a family of sets $\left(P_{r}\right)_{r \in S}=\left(J_{r}, K_{r}\right)_{r \in S} \subset$ $\mathbb{R} \times \mathbb{R}^{2}$ such that, for $\left(t^{\prime}, x^{\prime}\right) \in\left\{\left|e^{i t^{\prime} \square} f^{\prime}\left(x^{\prime}\right)\right| \geq 2 \lambda_{0}\right\}, K_{r}$ is a square of center $x^{\prime}$ with $\left|K_{r}\right|=$ $\left(\lambda_{0} / c A\right)^{2} \leq 1 / 16$ and $J_{r} \subset \mathbb{R}$ is a closed interval of center $t^{\prime}$ with $\left|J_{r}\right|=\lambda_{0} / c A \leq 1 / 4$. Also, $\left(P_{r}\right)_{r \in S}$ satisfies the following: for $(r, s) \in S \times S$ with $r \neq s, \operatorname{Int}\left(P_{r}\right) \cap \operatorname{Int}\left(P_{s}\right)=\emptyset$ and

$$
\left\{\left|e^{i t^{\prime} \square} f^{\prime}\left(x^{\prime}\right)\right| \geq 2 \lambda_{0}\right\} \subset \bigcup_{r \in S} P_{r} \subset\left\{\left|e^{i t^{\prime} \square} f^{\prime}\left(x^{\prime}\right)\right| \geq \lambda_{0}\right\}
$$

where $\operatorname{Int}\left(P_{r}\right)$ is the interior of $P_{r}$.

Let $N$ be the cardinality of $S$. Then, by the Strichartz inequality, $N$ is bounded. In fact,

$$
\begin{aligned}
N\left(\frac{\lambda_{0}}{c A}\right)^{3}=\left|\bigcup_{r \in S} P_{r}\right| & \leq\left|\left\{\left|e^{i t^{\prime} \square} f^{\prime}\left(x^{\prime}\right)\right| \geq \lambda_{0}\right\}\right| \\
& \leq \lambda_{0}^{-4}\left\|e^{i t^{\prime} \square} f^{\prime}\right\|_{L^{4}\left(\mathbb{R}^{3}\right)}^{4} \leq \lambda_{0}^{-4}\left\|f^{\prime}\right\|_{L^{2}}^{4}=\lambda_{0}^{-4}\|f\|_{L^{2}}^{4} .
\end{aligned}
$$

Since $\left\{\left|e^{i t^{\prime} \square} f^{\prime}\left(x^{\prime}\right)\right| \geq 2 \lambda_{0}\right\}$ is covered by $\left\{P_{n}\right\}_{1 \leq n \leq N}$,

$$
\int_{\mathbb{R}}\left(\int_{\bar{P}_{t}}\left|e^{i t^{\prime} \square} f^{\prime}\left(x^{\prime}\right)\right|^{r} d x^{\prime}\right)^{q / r} d t^{\prime}<\varepsilon^{q}
$$


where

$$
\bar{P}_{t}=\left\{x^{\prime} \in \mathbb{R}^{2}:\left(t^{\prime}, x^{\prime}\right) \in \mathbb{R}^{3} \mid \bigcup_{n=1}^{N} P_{n}\right\}
$$

For each $1 \leq n \leq N$, let $Q_{n}$ be the set

$$
\left\{(t, x):\left|x_{1}-2 \pi t \zeta_{2}-\frac{x_{1}^{n}}{a}\right|<\frac{1}{4 a},\left|x_{2}-2 \pi t \zeta_{1}-\frac{x_{2}^{n}}{b}\right|<\frac{1}{4 b},\left|t-\frac{t^{n}}{a b}\right|<\frac{1}{8 a b}\right\}
$$

where $\left(t^{n} ; x_{1}^{n}, x_{2}^{n}\right)=\left(t^{n} ; x^{n}\right)$ denotes the center of $P_{n}$. Let $\bar{Q}_{t}=\left\{x^{\prime} \in \mathbb{R}^{2}:\left(t^{\prime}, x^{\prime}\right) \in\right.$ $\left.\mathbb{R}^{3} \backslash \cup Q_{n}\right\}$. Then

$$
\begin{aligned}
\| e^{i t \square} & f \|_{L_{t}^{q} L_{x}^{r}\left(\mathbb{R}^{3} \backslash \cup Q_{n}\right)}^{q} \\
& =(a b)^{q / 2} \int_{\mathbb{R}}\left(\int_{\bar{Q}_{t}}\left|e^{i t^{\prime} \square} f^{\prime}\left(x^{\prime}\right)\right|^{r} d x\right)^{q / r} d t \\
& =(a b)^{q / 2} \int_{\mathbb{R}}\left(\int_{\bar{Q}_{t}}\left|e^{i a b t \square} f^{\prime}\left(a\left(x_{1}-2 \pi t \zeta_{2}\right), b\left(x_{2}-2 \pi t \zeta_{1}\right)\right)\right|^{r} d x\right)^{q / r} d t \\
& \leq(a b)^{q / 2} \int_{\mathbb{R}}\left(\int_{\bar{P}_{t}}\left|e^{i \bar{t} \square} f^{\prime}\left(\overline{x_{1}}, \bar{x}_{2}\right)\right|^{r} \frac{1}{a b} d \bar{x}\right)^{q / r} \frac{1}{a b} d \bar{t} \\
& =(a b)^{q / 2-(q / r+1)}\left\|e^{i \bar{t} \square} f^{\prime}\right\|_{L_{t}^{q} L_{x}^{r}\left(\mathbb{R}^{3} \backslash \cup P_{n}\right)}^{q}<(a b)^{q / 2-(q / r+1)} \varepsilon^{q} .
\end{aligned}
$$

Therefore, we may conclude that

$$
\left\|e^{i t \square} f\right\|_{L_{t}^{q} L_{x}^{r}\left(\mathbb{R}^{3} \backslash \cup Q_{n}\right)}<(a b)^{1 / 2-(1 / r+1 / q)} \varepsilon=\varepsilon .
$$

\section{Mass concentration phenomenon}

The following result implies Theorem 1.1, as was observed in Remarks 1.2 and 1.3. Theorem 4.1. Suppose that $u=u(t, x)$ is a solution to

$$
\left\{\begin{array}{l}
i u_{t}+\square u+\gamma|u|^{2} u=0 \\
u(0, x)=u_{0}(x) \in L^{2}\left(\mathbb{R}^{2}\right)
\end{array}\right.
$$

for some $\gamma \in \mathbb{R} \backslash\{0\}$. Let $(q, r)$ be an admissible pair with $q \leq r \leq 6$. Suppose that the solution satisfies $\|u\|_{L_{t}^{q} L_{x}^{r}\left([0, t) \times \mathbb{R}^{2}\right)}<\infty$ for $0<t<T_{\max }$ and that $\|u\|_{L_{t}^{q} L_{x}^{r}\left(\left[0, T_{\max }\right) \times \mathbb{R}^{2}\right)}=\infty$. Then

$$
\limsup _{t \nearrow T_{\max }} \sup _{\substack{\text { a rectangle } R \\|R| \leq\left(T_{\max }-t\right)}}\left(\int_{R}|u(t, x)|^{2} d x\right)^{1 / 2}>\varepsilon
$$

where $\varepsilon$ is a constant depending only on $\gamma$ and $\left\|u_{0}\right\|_{L^{2}\left(\mathbb{R}^{2}\right)}$. 
Proof. For a small fixed $\eta>0$, and for all times $T_{0}<T_{\max }$, there exists $T_{1}<T_{\max }$ such that $\|u\|_{L_{t}^{q} L_{x}^{r}\left(\left(T_{0}, T_{1}\right) \times \mathbb{R}^{2}\right)}=\eta$. By Duhamel's principle, for $t \in\left(T_{0}, T_{\max }\right)$,

$$
u_{t}(x)=e^{i\left(t-T_{0}\right) \square} u_{T_{0}}(x)+i \gamma \int_{T_{0}}^{t} e^{i(t-s) \square}|u(s)|^{2} u(s) d s .
$$

Step 1. Controlling the inhomogeneous part.

For any $t \in\left(T_{0}, T_{1}\right)$, let us set $F(u)=i \gamma \int_{T_{0}}^{t} e^{i(t-s) \square}|u(s)|^{2} u(s) d s$. It follows that

$$
\|F(u)\|_{L_{t}^{q} L_{x}^{r}\left(\left(T_{0}, T_{1}\right) \times \mathbb{R}^{2}\right)} \leq|\gamma| C\left\||u|^{2} u\right\|_{L_{t}^{\tilde{q}^{\prime}} L_{x}^{\tilde{r}^{\prime}}}=|\gamma| C\|u\|_{L_{t}^{q} L_{x}^{r^{r}}}^{3}=|\gamma| C \eta^{3}
$$

by (2.3) and Remark 1.3.

Hence, if we choose $\eta$ small enough such that

$$
\eta \leq\left(3^{q} 2\left((|\gamma| C)^{2}+1\right)\right)^{-1 / 4} \leq(1+|\gamma| C)^{-1 / 2},
$$

it follows that

$$
\left\|e^{i\left(t-T_{0}\right) \square} u_{T_{0}}\right\|_{L_{t}^{q} L_{x}^{r}\left(\left(T_{0}, T_{1}\right) \times \mathbb{R}^{2}\right)} \geq \eta-|\gamma| C \eta^{3} \geq \eta^{3} .
$$

Step 2. Decomposing the initial data.

We start with

$$
\begin{aligned}
\eta^{q} & =\int_{T_{0}}^{T_{1}}\left(\int_{\mathbb{R}^{2}}|u|^{r} d x\right)^{q / r} d t \\
& \leq 3^{q}\left(I+I I+\int_{T_{0}}^{T_{1}}\left(\int_{\mathbb{R}^{2}}|u|^{2}\left|\sum_{n=1}^{N_{0}} e^{i\left(t-T_{0}\right) \square} f_{n}\right|^{r-2} d x\right)^{q / r} d t\right)
\end{aligned}
$$

where

$$
\begin{gathered}
I=\int_{T_{0}}^{T_{1}}\left(\int_{\mathbb{R}^{2}}|u|^{2}\left|u(x, t)-e^{i\left(t-T_{0}\right) \square} u_{T_{0}}\right|^{r-2} d x\right)^{q / r} d t \\
I I=\int_{T_{0}}^{T_{1}}\left(\int_{\mathbb{R}^{2}}|u|^{2}\left|e^{i\left(t-T_{0}\right) \square} u_{T_{0}}-\sum_{n=1}^{N_{0}} e^{i\left(t-T_{0}\right) \square} f_{n}\right|^{r-2} d x\right)^{q / r} d t
\end{gathered}
$$

and $\left\{f_{n}\right\}_{n=1}^{N_{0}}$ is as in the proof of Lemma 3.7 below.

Using Hölder's inequality with $r / 2$ and $r /(r-2)$, we estimate

$$
\begin{aligned}
I & =\int_{T_{0}}^{T_{1}}\left(\int_{\mathbb{R}^{2}}|u|^{2}|F(u)|^{r-2} d x\right)^{q / r} d t \\
& \leq \int_{T_{0}}^{T_{1}}\left(\left(\int_{\mathbb{R}^{2}}|u|^{r} d x\right)^{2 / r}\left(\int_{\mathbb{R}^{2}}|F(u)|^{r} d x\right)^{(r-2) / r}\right)^{q / r} d t \\
& =\int_{T_{0}}^{T_{1}}\left(\int_{\mathbb{R}^{2}}|u|^{r} d x\right)^{2 q / r^{2}}\left(\int_{\mathbb{R}^{2}}|F(u)|^{r} d x\right)^{q(r-2) / r^{2}} d t
\end{aligned}
$$




$$
\begin{aligned}
& \leq\left(\int_{T_{0}}^{T_{1}}\left(\int_{\mathbb{R}^{2}}|u|^{r} d x\right)^{q / r} d t\right)^{2 / r}\left(\int_{T_{0}}^{T_{1}}\left(\int_{\mathbb{R}^{2}}|F(u)|^{r} d x\right)^{q / r} d t\right)^{(r-2) / r} \\
& =\|u\|_{L_{t}^{q} L_{x}^{r}}^{2 q / r}\|F(u)\|_{L_{t}^{q} L_{x}^{r}}^{q(r-2) / r} \leq \eta^{2 q / r}\left(|\gamma| C \eta^{3}\right)^{q(r-2) / r}=(|\gamma| C)^{2} \eta^{q+4}
\end{aligned}
$$

because of the fact that $3 q-4 q / r=q+4$.

Similarly, by Lemma 3.7,

$$
I I \leq\|u\|_{L_{t}^{q} L_{x}^{r}}^{2 q / r}\left\|e^{i\left(t-T_{0}\right) \square} u_{T_{0}}-\sum_{n=1}^{N_{0}} e^{i\left(t-T_{0}\right) \square} f_{n}\right\|_{L_{t}^{q} L_{x}^{r}}^{q(r-2) / r} \leq \eta^{2 q / r}\left(\eta^{3}\right)^{q(r-2) / r}=\eta^{q+4} .
$$

Therefore, by (4.1),

$$
\int_{T_{0}}^{T_{1}}\left(\int_{\mathbb{R}^{2}}|u|^{2}\left|\sum_{n=1}^{N_{0}} e^{i\left(t-T_{0}\right) \square} f_{n}\right|^{r-2} d x\right)^{q / r} d t \geq \frac{\eta^{q}}{3^{q} 2} .
$$

Then there exists an integer $n_{0}$ between 1 and $N_{0}$ and a function $\hat{f}_{0}=\hat{f}_{n_{0}}$ such that for some $\varepsilon_{0}>0$,

$$
\int_{T_{0}}^{T_{1}}\left(\int_{\mathbb{R}^{2}}|u|^{2}\left|e^{i\left(t-T_{0}\right) \square} f_{0}\right|^{r-2} d x\right)^{q / r} d t \geq \varepsilon_{0}
$$

where $\hat{f}_{0}$ is supported in $R$ and $\left|\hat{f}_{0}\right| \leq A|R|^{-1 / 2}$ from Lemma 3.7.

Step 3. Figuring out the concentration region.

From Lemma 3.9, we can show that there exist an integer $N_{1}$ and a set of rectangles $\left\{Q_{n}\right\}_{1 \leq n \leq N_{1}}$, where $Q_{n}=\left\{(t, x) \in \mathbb{R}^{3}:\left(x_{1}-2 \pi t \zeta_{2}, x_{2}-2 \pi t \zeta_{1}\right) \in R_{n}, t \in I_{n}\right\}$, $R_{n}$ is a rectangle of measure $|R|^{-1}$, and $I_{n}$ is an interval of length $|R|^{-1}$ such that

$$
\left\|e^{i\left(t-T_{0}\right) \square} f_{0}\right\|_{L_{t}^{q} L_{x}^{r}\left(\mathbb{R} \times \mathbb{R}^{2} \backslash \cup_{n=1}^{N_{1}} Q_{n}\right)}<\left(\frac{\varepsilon_{0}}{2 \eta^{2 q / r}}\right)^{r / q(r-2)} .
$$

By Hölder's inequality with $2 / r+(r-2) / r=1$, on

$$
\tilde{Q}_{t}=\left\{x \in \mathbb{R}^{2}:(t, x) \in\left(\left(T_{0}, T_{1}\right) \times \mathbb{R}^{2}\right) \mid \bigcup_{n=1}^{N_{1}} Q_{n}\right\},
$$

we have

$$
\begin{aligned}
& \int_{T_{0}}^{T_{1}}\left(\int_{\tilde{Q}_{t}}|u|^{2}\left|e^{i\left(t-T_{0}\right) \square} f_{0}\right|^{r-2} d x\right)^{q / r} d t \\
& \quad \leq\|u\|_{L_{t}^{q} L_{x}^{r}}^{2 q / r}\left\|e^{i\left(t-T_{0}\right) \square} f_{0}\right\|_{L_{t}^{q} L_{x}^{r}\left(\left(\left(T_{0}, T_{1}\right) \times \mathbb{R}^{2}\right) \backslash \cup_{n=1}^{N_{1}} Q_{n}\right)}^{q(r-)} \\
& \quad<\eta^{2 q / r}\left(\frac{\varepsilon_{0}}{2 \eta^{2 q / r}}\right)=\frac{\varepsilon_{0}}{2} .
\end{aligned}
$$


It follows that, on $\left(\left(T_{0}, T_{1}\right) \times \mathbb{R}^{2}\right) \cap\left(\bigcup_{n=1}^{N_{1}} Q_{n}\right)$,

$$
\int_{T_{0}}^{T_{1}}\left(\int_{\tilde{Q}^{t}}|u|^{2}\left|e^{i\left(t-T_{0}\right) \square} f_{0}\right|^{r-2} d x\right)^{q / r} d t \geq \frac{\varepsilon_{0}}{2}
$$

where

$$
\tilde{Q}^{t}=\left\{x \in \mathbb{R}^{2}:(t, x) \in\left(\left(T_{0}, T_{1}\right) \times \mathbb{R}^{2}\right) \cap\left(\bigcup_{n=1}^{N_{1}} Q_{n}\right)\right\} .
$$

Hence there exists a rectangle $Q_{0}=R_{0} \times I_{0} \in\left\{Q_{n}\right\}_{n=1}^{N_{1}}$ such that

$$
\int_{\left(T_{0}, T_{1}\right) \cap I_{0}}\left(\int_{Q_{0}^{t}}|u(x, t)|^{2}\left|e^{i\left(t-T_{0}\right) \square} f_{0}\right|^{r-2} d x\right)^{q / r} d t \geq \frac{\varepsilon_{0}}{2 N_{1}}=: \varepsilon_{1}
$$

where $Q_{0}^{t}=\left\{x \in \mathbb{R}^{2}:\left(x_{1}-2 \pi t \zeta_{2}, x_{2}-2 \pi t \zeta_{1}\right) \in R_{0}, t \in I_{0}\right\}, R_{0}$ is a rectangle of measure $|R|^{-1}$ and $I_{0}$ is an interval of length $|R|^{-1}$.

Step 4. Determining the size of windows.

Since $\left|\hat{f}_{0}\right| \leq A|R|^{-1 / 2}$ and $\hat{f}_{0}$ is supported in $R$,

$$
\left|e^{i\left(t-T_{0}\right) \square} f_{0}\right| \leq \int_{R}|\hat{f}| \leq|R| A|R|^{-1 / 2}=A|R|^{1 / 2},
$$

and

$$
\begin{aligned}
\varepsilon_{1} & \leq \int_{\left(T_{0}, T_{1}\right) \cap I_{0}}\left(\int_{Q_{0}^{t}}|u(t, x)|^{2}\left|e^{i\left(t-T_{0}\right) \square} f_{0}\right|^{r-2} d x\right)^{q / r} d t \\
& \leq\left(A|R|^{1 / 2}\right)^{q(r-2) / r} \int_{\left(T_{0}, T_{1}\right) \cap I_{0}}\left(\int_{Q_{0}^{t}}|u(t, x)|^{2} d x\right)^{q / r} d t \\
& \leq\left(A|R|^{1 / 2}\right)^{q(r-2) / r}\left(T_{1}-T_{0}\right)\left\|u_{0}\right\|_{L^{2}\left(\mathbb{R}^{2}\right)}^{2 q / r}
\end{aligned}
$$

Thus

$$
T_{1}-T_{0} \geq \frac{\varepsilon_{1}}{\left(A|R|^{1 / 2}\right)^{q(r-2) / r}\left\|u_{0}\right\|_{L^{2}\left(\mathbb{R}^{2}\right)}^{2 q / r}}=: \theta
$$

We can observe that

$$
\int_{T_{1}-\frac{1}{2} \theta}^{T_{1}}\left(\int_{Q_{0}^{t}}|u(t, x)|^{2}\left|e^{i\left(t-T_{0}\right) \square} f_{0}\right|^{r-2} d x\right)^{q / r} d t \leq \frac{1}{2} \theta\left(A|R|^{1 / 2}\right)^{q(r-2) / r}\left\|u_{0}\right\|_{L^{2}}^{2 q / r}=\frac{\varepsilon_{1}}{2} .
$$

So

$$
\begin{aligned}
\frac{\varepsilon_{1}}{2} & \leq \int_{\left(T_{0}, T_{1}-\frac{1}{2} \theta\right) \cap I_{0}}\left(\int_{Q_{0}^{t}}|u|^{2}\left|e^{i\left(t-T_{0}\right) \square} f_{0}\right|^{r-2}\right)^{q / r} d t \\
& \leq\left|I_{0}\right| \sup _{t \in\left(T_{0}, T_{1}-\frac{1}{2} \theta\right)}\left(\int_{Q_{0}^{t}}|u|^{2}\left|e^{i\left(t-T_{0}\right) \square} f_{0}\right|^{r-2}\right)^{q / r} \\
& \leq|R|^{-1}\left(A|R|^{1 / 2}\right)^{q(r-2) / r}\left(\sup _{t \in\left(T_{0}, T_{1}-\frac{1}{2} \theta\right)} \int_{Q_{0}^{t}}|u|^{2} d x\right)^{q / r} .
\end{aligned}
$$


Then we can say that

$$
\sup _{t \in\left(T_{0}, T_{1}-\frac{1}{2} \theta\right)}\left(\int_{Q_{0}^{t}}|u|^{2} d x\right)^{q / r} \geq \frac{\varepsilon_{1}}{2 A^{q(r-2) / r}},
$$

or

$$
\sup _{t \in\left(T_{0}, T_{1}-\frac{1}{2} \theta\right)} \int_{Q_{0}^{t}}|u|^{2} d x \geq C\left(\frac{\varepsilon_{1}}{2}\right)^{r / q}
$$

where $C=1 / A^{r-2}$.

Therefore, for all $T_{0}<T_{\max }$, there exist $t_{0} \in\left(T_{0}, T_{1}-1 / 2 \theta\right)$ and a rectangle $Q_{0}^{t_{0}}$ such that

$$
\int_{Q_{0}^{t_{0}}}\left|u\left(t_{0}, x\right)\right|^{2} d x>\frac{C}{4}\left(\frac{\varepsilon_{1}}{2}\right)^{r / q} .
$$

Note that

$$
t_{0} \leq T_{\max }-\frac{1}{2} \theta=T_{\max }-\frac{\varepsilon_{2}}{|R|^{q(r-2) / 2 r}}
$$

where $\varepsilon_{2}=\varepsilon_{1}\left(2 A^{q(r-2) / r}\left\|u_{0}\right\|_{L^{2}}^{2 q / r}\right)^{-1}$.

Because $(q, r)$ is an admissible pair, $q(r-2) / 2 r=q / 2-q / r=1$. Then

$$
\left|Q_{0}^{t_{0}}\right|=\frac{1}{|R|} \leq \frac{1}{\varepsilon_{2}}\left(T_{\max }-t_{0}\right) .
$$

Dividing $Q_{0}^{t_{0}}$ into $m=\left\lceil 1 / \varepsilon_{2}\right\rceil$ rectangles, there exists a rectangle $R^{\prime}$ such that $\left|R^{\prime}\right| \leq T_{\max }-t_{0}$. Therefore,

$$
\int_{R^{\prime}}\left|u\left(t_{0}, x\right)\right|^{2} d x>\frac{C}{4 m}\left(\frac{\varepsilon_{1}}{2}\right)^{r / q}=: \varepsilon_{3} .
$$

Step 5. Conclusion.

We consider a sequence $\left\{T_{n}\right\}$ such that $0=T_{1}<T_{2}<\cdots<T_{n}<T_{n+1}<\cdots<T_{\max }$ and $\|u\|_{L_{t}^{q} L_{x}^{r}\left(\left(T_{n}, T_{n+1}\right) \times \mathbb{R}^{2}\right)}=\eta$. For each interval $\left(T_{n}, T_{n+1}\right)$, there exist $t_{n} \in\left(T_{n}, T_{n+1}\right)$ such that

$$
\sup _{\substack{\text { a rectangle } R \\|R| \leq\left(T_{\max }-t_{n}\right)}}\left(\int_{R}\left|u\left(t_{n}, x\right)\right|^{2} d x\right)^{1 / 2}>\sqrt{\varepsilon_{3}}
$$

by (4.2). Thus we get a sequence $\left\{t_{n}\right\}$ of time such that $t_{n} \rightarrow T_{\max }$ as $n \rightarrow \infty$. This gives the conclusion of the theorem with $\varepsilon=\sqrt{\varepsilon_{3}}$.

\section{Acknowledgements}

We would like to thank the referee for comments on the manuscript. We thank also Jong-Guk Bak and Sanghyuk Lee for very helpful comments. 


\section{References}

[1] P. Bégout and A. Vargas, 'Mass concentration phenomena for the $L^{2}$-critical nonlinear Schrödinger equation', Trans. Amer. Math. Soc. 359 (2007), 5257-5282.

[2] J. Bourgain, 'Refinements of Strichartz' inequality and applications to 2D-NLS with critical monlinearity', Int. Math. Res. Not. 5 (1998), 253-283.

[3] T. Cazenave, Semilinear Schrödinger Equations, Courant Lecture Notes in Mathematics, 10 (New York University, Courant Institute of Mathematical Sciences, New York, 2003).

[4] M. Chae, S. Hong, J. Kim, S. Lee and C. W. Yang, 'On mass concentration for the $L^{2}$-critical nonlinear Schrödinger equations', Comm. Partial Differential Equations 34(4-6) (2009), 486-505.

[5] M. Chae, S. Hong and S. Lee, 'Mass concentration for the $L^{2}$-critical nonlinear Schrödinger equations of higher orders', Discrete Contin. Dyn. Syst. 29(3) (2011), 909-928.

[6] M. Christ and A. Kiselev, 'Maximal functions associated to filtrations', J. Funct. Anal. 179(2) (2001), 409-425.

[7] M. Keel and T. Tao, 'Endpoint Strichartz estimates', Amer. J. Math. 120 (1998), 955-980.

[8] S. Lee, 'Bilinear restriction estimates for surfaces with curvatures of different signs', Trans. Amer. Math. Soc. 358 (2006), 3511-3533.

[9] S. Lee, K. Rogers and A. Vargas, 'Sharp null form estimates for the wave equation in $\mathbb{R}^{3+1}$, Int. Math. Res. Not. IMRN (2008), Art. ID rnn 096, 18 pp.

[10] S. Lee and A. Vargas, 'Sharp null form estimates for the wave equation', Amer. J. Math. 130(5) (2008), 1279-1326.

[11] A. Moyua, A. Vargas and L. Vega, 'Restriction theorems and maximal operators related to oscillatory integrals in $\mathbb{R}^{3}$, Duke Math. J. 96 (1999), 547-574.

[12] K. Rogers and A. Vargas, 'A refinement of the Strichartz inequlity on the saddle and application', J. Funct. Anal. 241(1) (2006), 212-231.

[13] C. Sulem and P.-L. Sulem, The Nonlinear Schrödinger Equation, Applied Mathematical Sciences, 139 (Springer, New York, 1999).

[14] T. Tao, 'A sharp bilinear restriction estimate for paraboloids', Geom. Funct. Anal. 13 (2003), 1359-1384.

[15] T. Tao, Nonlinear Dispersive Equations, CBMS Regional Conference Series in Mathematics, 106 (American Mathematical Society, Providence, RI, 2006).

[16] T. Tao, A. Vargas and L. Vega, 'A bilinear approach to the restriction and Kakeya conjecture', J. Amer. Math. Soc. 11 (1998), 967-1000.

[17] A. Vargas, 'Restriction theorems for a surface with negative curvature', Math. Z. 249(1) (2005), 97-111.

SEHEON HAM, Department of Mathematics, Pohang University of Science and Technology, Pohang 790-784, Korea

e-mail: beatles8@postech.ac.kr 